\title{
One-Dimensional Multiple-Temperature Gas-Kinetic Bhatnagar- Gross-Krook Scheme for Shock Wave Computation
}

\author{
Chunpei Cai* and Danny D. Liu \\ ZONA Technology, Scottsdale, Arizona 85258 \\ and \\ Kun $\mathrm{Xu}$ \\ Hong Kong University of Science and Technology, Kowloon, Hong Kong \\ DOI: $\underline{10.2514 / 1.27432}$
}

\begin{abstract}
Accurately computing the inner structure of normal shock waves or oblique shock waves is crucial for many hypersonic applications. As such, it will improve the prediction accuracy of aerodynamics properties and aerothermal effects on hypersonic vehicles and spacecraft during atmospheric entries. Because a shock wave usually has a thickness of a few mean free paths, it is very difficult to accurately compute the detailed nonequilibrium inner structure across a shock wave with a continuum method. This paper reports a gas-kinetic Bhatnagar-Gross-Krook scheme for computations of one-dimensional vibrationally nonequilibrium nitrogen flows through a planar shock wave. The present gas-kinetic Bhatnagar-Gross-Krook scheme solves for the shock structure with multiple temperatures, including two translational temperatures, one rotational temperature, and one vibrational temperature. The salient features of the present gas-kinetic Bhatnagar-Gross-Krook method are multifold. Its applicability covers a wide simulation regime, extending that of continuum flows to the transition flows; it is more computationally efficient in time than the traditional direct simulation Monte Carlo method for shock wave simulation. To provide proper downstream subsonic boundary conditions for very strong shock waves, it is required to determine a proper postshock equilibrium state in which all temperatures have accomplished relaxation processes to a common equilibrium temperature. Analytical expressions of a complete set of generalized Rankine-Hugoniot relations across a planar shock wave are obtained to account for the variant specific heat ratio due to inner energy excitations. Numerical simulation results by the present gas-kinetic Bhatnagar-Gross-Krook scheme and the direct simulation Monte Carlo method are found to be in good agreement.
\end{abstract}

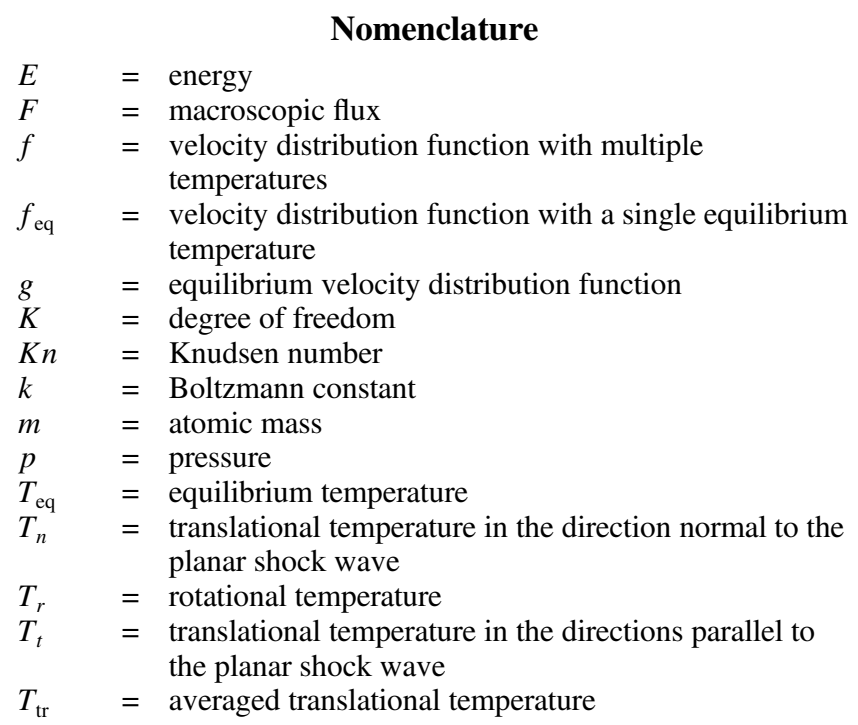

Presented as Paper 2006-7900 at the 14th AIAA/AHI Space Planes and Hypersonic Systems and Technologies Conference, Canberra, Australia, 811 November 2006; received 22 August 2006; revision received 31 August 2007; accepted for publication 5 January 2008. Copyright $@ 2008$ by Chunpei Cai. Published by the American Institute of Aeronautics and Astronautics, Inc., with permission. Copies of this paper may be made for personal or internal use, on condition that the copier pay the $\$ 10.00$ per-copy fee to the Copyright Clearance Center, Inc., 222 Rosewood Drive, Danvers, MA 01923; include the code 0001-1452/08 \$10.00 in correspondence with the CCC.

${ }^{*}$ Computational Fluid Dynamics Specialist, 9489 East Ironwood Square Drive. Senior Member AIAA.

${ }^{\dagger}$ President, 9489 East Ironwood Square Drive. Fellow AIAA.

Frofessor, Department of Mathematics, Clear Water Bay. Member AIAA.

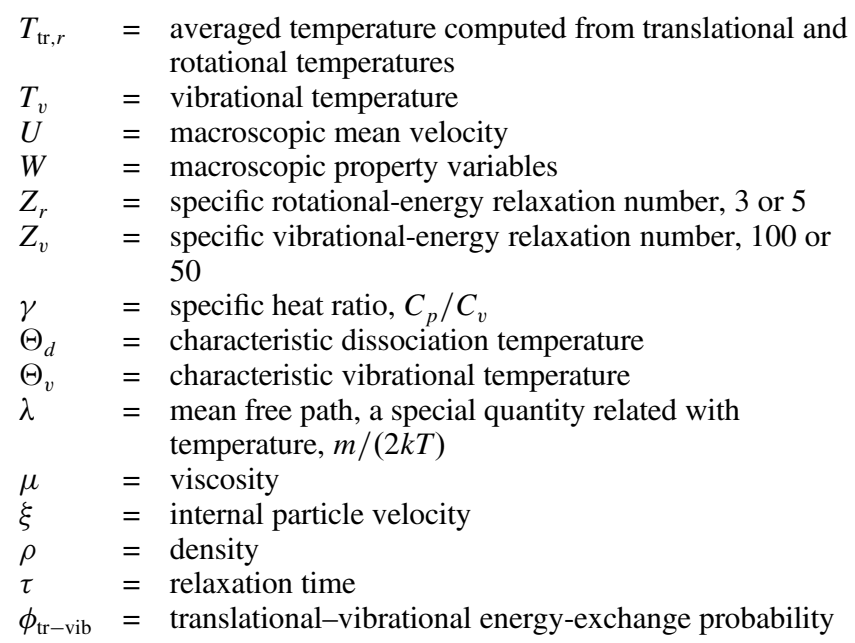

\section{Introduction}

$\mathbf{H}$ YPERSONIC rarefied gas dynamics for practical aerothermodynamic consideration often lie in the transition regime in which the Knudsen number range is on the order of 0.01-10. In this regime, hypersonic rarefied airflows are characterized by large nonequilibrium regions with multiple temperatures, including different translational temperatures $T_{n}$ and $T_{t}$, rotational temperature $T_{r}$, and vibrational temperature $T_{v}$. Several approaches have been used in an attempt to solve this problem. The direct simulation Monte Carlo (DSMC) method can easily model the nonequilibrium physics, but it becomes increasingly expensive as the Knudsen number becomes closer to the lower end of the transition region. On the other hand, the classical continuum solution using computational fluid dynamics (CFD) is found to deteriorate as the flow is approaching this transition regime. Directly solving the Boltzmann equation [1] by retaining the complete collision integral is hampered 
by the difficulty in the incorporation of the nonequilibrium physics with rotational and vibrational energy and the extremely expensive simulation cost.

The Bhatnagar-Gross-Krook (BGK) model of the Boltzmann equation appears to provide a viable method to solve for the nonequilibrium flow problem because of its ease of formulation in the multitemperature modeling of the distribution functions. But the current BGK approaches are mostly confined to flows of low Mach number. Among them, one gas-kinetic BGK method proposed by $\mathrm{Xu}$ and Josyula [2] and $\mathrm{Xu}[3,4]$ stands out as a promising approach to compute hypersonic nonequilibrium flows with shock waves. The primary outstanding features of the BGK method are described in a review paper [4].

The success of the BGK scheme for the rarefied flow computation is due to the following. In the traditional Chapman-Enskog expansion, the real-gas distribution function is obtained by an expansion around an equilibrium state (i.e., the so-called Maxwellian distribution). However, for a highly nonequilibrium flow, because the gas distribution function may be far away from the equilibrium, the direct expansion becomes problematic, such as the intrinsic instability of the Burnett and super-Burnett equations at high Knudsen numbers. In our model, we use two processes to describe the nonequilibrium state. First, we apply the Chapman-Enskog expansion directly into a nonequilibrium multiple-temperature state (see Fig. 1). Then the multiple-temperature model will relax to an equilibrium state, and the relaxation process depends on the relative relaxation time. For the rotational and vibrational modes, it takes a long time to relax to a Maxwellian distribution, and the real-gas distribution function may stay forever in a nonequilibrium state.

The major part of the present work is an extension of the gaskinetic BGK scheme in that it is based on early work by $\mathrm{Xu}$ and Josyula [2] and $\mathrm{Xu}$ [3]. We propose a complete gas-kinetic BGK scheme before flow dissociation for the computation of a planar shock wave with all possible multiple temperatures: namely, two translational temperatures $T_{n}$ and $T_{t}$, one rotational temperature $T_{r}$, and one vibrational temperature $T_{v}$. To validate this new scheme, three test cases of strong nitrogen shock waves with $M_{\infty}=5.0,10.0$, 15.0 are simulated with the BGK scheme and the DSMC method. For both the BGK scheme and the DSMC method, proper downstream equilibrium boundary conditions are required for the simulation. However, due to the consideration of the vibrational energy, the specific heat ratio $\gamma=C_{p} / C_{v}$ is variant across the shock waves. Hence, the classical Rankine-Hugoniot relations are not applicable. To address this problem, we derived a set of generalized RankineHugoniot relations with which the proper downstream flow conditions can be determined for the DSMC and BGK simulations.

\section{One-Dimensional Thermal Nonequilibrium Multiple-Temperature Kinetic BGK Model}

In this section, we propose a general one-dimensional gas-kinetic BGK scheme to compute nonequilibrium flows with multiple temperatures. The model is applicable in simulations of planar shock waves with monatomic, diatomic, and polyatomic molecules.

The Boltzmann equation expresses the behavior of a manyparticle kinetic system in terms of the evolution equation for a singleparticle gas distribution function. The right-hand side of the Boltzmann equation is mainly two body collisions that are valid for a large range of pressure, from several hundreds of atmospheres to free-molecular flow [5]. To simplify the Boltzmann equation, the one-dimensional BGK model is formulated as

$$
\frac{\partial f}{\partial t}+u \frac{\partial f}{\partial x}=\frac{g-f}{\tau}+Q
$$

where $f$ is the normalized number density distribution of molecules at position $x$ with a particle velocity $u$ at time $t, \tau=\mu / p$ is the characteristic relaxation time ( $\mu$ can be computed by Sutherland's law or by a power law), $Q$ represents the relaxation process to a Maxwellian distribution, and $g$ is the equilibrium state of the gas with the following specific expression:

$$
\begin{aligned}
g= & \rho\left(\frac{\lambda_{x}}{\pi}\right)^{1 / 2} \exp \left(-\lambda_{x}(u-U)^{2}\right)\left(\frac{\lambda_{t}}{\pi}\right)^{K_{t} / 2} \\
& \times \exp \left(-\lambda_{t} \xi_{t}^{2}\right)\left(\frac{\lambda_{r}}{\pi}\right)^{K_{r} / 2} \exp \left(-\lambda_{r} \xi_{r}^{2}\right)\left(\frac{\lambda_{v}}{\pi}\right)^{K_{v} / 2} \exp \left(-\lambda_{v} \xi_{v}^{2}\right)
\end{aligned}
$$

where $\rho$ is the density, $U$ is the macroscopic fluid velocity along the $x$ direction, $\lambda=m /(2 k T), m$ is the molecular mass, $k$ is the Boltzmann constant, and $T$ is a specific temperature. For an equilibrium flow, an internal variable $\xi$ accounts for the tangential, rotational, and vibrational modes and has an expression of $\xi^{2}=\xi_{t}^{2}+\xi_{r}^{2}+\xi_{v}^{2}$, in which $\xi_{t}, \xi_{r}$, and $\xi_{v}$ have $K_{t}, K_{r}$, and $K_{v}$ degrees of freedom of translational (in tangential directions), rotational, and vibrational energy. For nitrogen, $K_{t}=2, K_{r}=2$, and $K_{v}$ are determined by the vibrational-energy equation (19). Notice that the specific heat ratio $\gamma$ is not constant for vibrational nonequilibrium flows, and the BGK solver must compute it locally in each time step for each cell:

$$
\gamma=\frac{3+K_{r}+K_{v}+2}{3+K_{r}+K_{v}}
$$

The mass $\rho$, momentum $\rho U$, total energy $\rho E$, thermal energy along the tangential directions $\rho E_{t}$, rotational energy $\rho E_{r}$, and vibrational energy $\rho E_{v}$ at each cell center are the moments of $f$ :

$$
W=\left(\rho, \rho U, \rho E, \rho E_{t}, \rho E_{r}, \rho E_{v}\right)^{T}=\int \Psi f \mathrm{~d} u \mathrm{~d} \xi_{t} \mathrm{~d} \xi_{r} \mathrm{~d} \xi_{v}
$$

where $\Psi$ has the components

$$
\Psi=\left(1, u, \frac{1}{2}\left(u^{2}+\xi_{t}^{2}+\xi_{r}^{2}+\xi_{v}^{2}\right), \frac{1}{2} \xi_{t}^{2}, \frac{1}{2} \xi_{r}^{2}, \frac{1}{2} \xi_{v}^{2}\right)^{T}
$$

and $\mathrm{d} u \mathrm{~d} \xi_{t} \mathrm{~d} \xi_{r} \mathrm{~d} \xi_{v}$ is the volume element in phase space. Because only mass, momentum, and total energy are conserved during particle collisions, the collision term on the right-hand side of the BGK model equation satisfies the following collision condition:

$$
\int Q \Psi \mathrm{d} u \mathrm{~d} \xi_{t} \mathrm{~d} \xi_{r} \mathrm{~d} \xi_{v}=S=\left(0,0,0, s_{t}, s_{r}, s_{v}\right)^{T}
$$

In this scheme, the source terms are modeled as $s_{t}=$ $\rho R\left(T_{t}-0.5\left(T_{\mathrm{tr}}+T_{\mathrm{tr}, r}\right)\right) / \tau, \quad s_{r}=\rho R\left(T_{r}-T_{\mathrm{tr}}\right) /\left(Z_{r} \tau\right), \quad s_{v}=$ $\rho\left(R T_{v}-e_{v}\left(T_{\mathrm{tr}}\right)\right) /\left(Z_{v} \tau\right), T_{r}, T_{v}, T_{\mathrm{tr}}$, and $T_{\mathrm{tr}, r}$ are internal rotational, vibrational, averaged translational, and averaged translational and rotational temperatures, respectively. $Z_{r}$ and $Z_{v}$ are the relaxation parameters for rotational and vibrational temperatures. Notice that in this scheme we assume that the rotational and vibrational temperatures relax toward the equilibrium translational temperature, based on the fact that the internal rotational and vibrational energy is obtained via energy exchange with the translational energy.

Base on the preceding BGK model, the Navier-Stokes equations can be derived with the Chapman-Enskog expansion with the firstorder expression only [4]]:

$$
f=g-\tau(\partial g / \partial t+u \partial g / \partial x)+\tau Q
$$

To provide the gradients for $g, \rho, U$, and $\lambda$ are assumed to be functions of both time and space [4]. The process to compute the slope in the equilibrium distribution $\bar{g}$ in Eq. (7) is quite complex, and the final format is shown in the Appendix. Note that the solutions in the Appendix are applicable to other situations such as monatomic flows when setting the coefficients $a_{5}$ and $a_{6}$ to zero. They are also applicable to study the rotational-temperature relaxation-only process by setting $a_{6}$ to zero.

An operator-splitting method will be used to solve Eq. (1). The general solution of $f$ in the BGK model with the inclusion of the $(g-f) / \tau$ term of Eq. (1) at a cell interface $x_{j+1 / 2}$ and time $t$ is 


$$
\begin{aligned}
& f\left(x_{j+1 / 2}, t, u, \xi_{t}, \xi_{r}, \xi_{v}\right) \\
& \quad=\frac{1}{\tau} \int_{0}^{t} g\left(x^{\prime}, t^{\prime}, u^{\prime}, \xi_{t}^{\prime}, \xi_{r}^{\prime}, \xi_{v}^{\prime}\right) \exp \left(-\left(t-t^{\prime}\right) / \tau\right) \mathrm{d} t^{\prime} \\
& \quad+\exp (-t / \tau) f_{0}\left(x_{j+1 / 2}-u t\right)
\end{aligned}
$$

where $x=x_{j+1 / 2}-u\left(t-t^{\prime}\right)$ is the particle trajectory, and $f_{0}$ is the initial gas distribution function at the beginning of each time step $(t=0)$. How to properly construct $f_{0}$ was explained in [4]. The preceding solution essentially combines the particle movement and particle collision effects into one procedure step, and hence viscous and inviscid fluxes are computed in one procedure step as well. This is one of the fundamental advantages for the gas-kinetic BGK scheme. For the $Q$ term contribution in Eq. (1), it generates a gas distribution function based on a real Maxwellian distribution, which can be denoted as $f_{\text {eq }}$, where $f_{\text {eq }}$ is obtained as Eq. ( $\left.\underline{8}\right)$ with the replacement of $g$ by a real equal-temperature Maxwellian distribution.

To validate the preceding model, a finite volume method is used to solve the BGK model $[2,3]$ :

$$
W_{j}^{n+1}=W_{j}^{n}+\frac{1}{\Delta x} \int_{0}^{\delta t}\left[F_{j-1 / 2}(t)-F_{j+1 / 2}(t)\right] \mathrm{d} t+S_{j}^{n} \delta t
$$

where $W_{j}^{n}$ are the macroscopic properties, shown as Eq. (4), in the $j$ th cell center, and $F_{j+1 / 2}$ are the corresponding fluxes at a cell interface by solving the Boltzmann equation. In this scheme, the flux is computed by the following format [2]:

$$
F_{j+1 / 2}=\int \frac{1}{2} u \Psi\left(f+f_{\mathrm{eq}}\right)_{j+1 / 2} \mathrm{~d} u \mathrm{~d} \xi_{t} \mathrm{~d} \xi_{r} \mathrm{~d} \xi_{v}
$$

where $\delta t$ is the time step $\delta t=t^{n+1}-t^{n}$. In Eq. ( $\left.\underline{9}\right), S_{j}^{n}$ is the source term in the thermal-energy equations. The evaluation of the fluxes is based on the gas distribution functions $f$ and $f_{\text {eq }}$ at a cell interface, $f$ is a multiple-temperature distribution function, and $f_{\text {eq }}$ is a onetemperature distribution function. The evaluation of the flux by averaging with an equilibrium state velocity distribution function $f_{\text {eq }}$ is proved positive to obtain a smooth density profile across strong shock waves [2]. Schematically, it basically shows that the preceding flux function is evaluated at the triangular point in Fig. 1 .

For the computations of the inner structures for a planar shock wave, the temperature profiles are the major concerns. Hence, a correct heat-flux relation is crucial for the computation. It is well known that the BGK model incorrectly provides a Prandtl number, $\operatorname{Pr}=1$, after taking moments to obtain the corresponding NavierStokes equations. Hence, the heat flux in the energy flux is not correct:

$q=\frac{1}{2} \int(u-U)\left[(u-U)^{2}+\xi_{t}^{2}+\xi_{r}^{2}+\xi_{v}^{2}\right] \frac{f+f_{\mathrm{eq}}}{2} \mathrm{~d} u \mathrm{~d} \xi_{t} \mathrm{~d} \xi_{r} \mathrm{~d} \xi_{v}$

The simplest and also most effective treatment is the Prandtl number fix [4], which is used in this study as well:

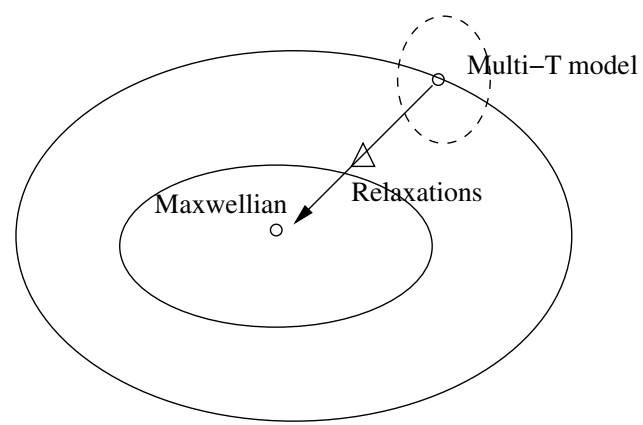

Fig. 1 Two processes to describe a nonequilibrium state: ChapmanEnskog expansion around a multiple-temperature state and a relaxation process to a Maxwellian distribution.

$$
F_{E}^{\text {new }}=F_{E}+\left(\frac{1}{P r}-1\right) q
$$

where $F_{E}$ is the energy flux in Eq. (10). This Prandtl-number-fix treatment has been successfully applied to many problems with correct heat-flux results [6].

Another important issue is to determine the the characteristic relaxation time step $\tau$. In this study, we adopt the following format to compute the parameter $\tau$ for each cell at each time step [4]:

$$
\tau=\frac{\mu}{p}+\frac{\left|\rho_{l} / \lambda_{l}-\rho_{r} / \lambda_{r}\right|}{\left|\rho_{l} / \lambda_{l}+\rho_{r} / \lambda_{r}\right|} \Delta t
$$

where subscripts 1 and $r$ represent quantities at the left and right cell center, and $\Delta t$ is the Courant-Friedrichs-Lewy (CFL) time step. The second part corresponds to the numerical viscosity. For strongshock-wave computations, the specific relaxation time $\tau$ must consider gradient terms. In this study, we adopted the generalized collision time $\tau_{*}$ introduced by $\mathrm{Xu}$ [7]. It is closely related to the collision time $\tau$, which is well-defined in the continuum flow regime:

$$
\tau_{*}=\frac{\tau}{1+\tau\left(\left\langle D^{2} f_{\text {eq }}\right\rangle /\left\langle D f_{\text {eq }}\right\rangle\right)}
$$

where $\left\langle D^{2} f_{\text {eq }}\right\rangle$ and $\left\langle D f_{\text {eq }}\right\rangle$ represent averaged moments with the distribution function $f_{\mathrm{eq}}$ [7]. In such, the higher-order gradient terms are introduced into the characteristic relaxation time, and some previous results indicate that it can effectively improve the computation results for the inner structures of strong shock waves.

\section{Post-Strong-Shock Equilibrium State Computation}

To test the preceding multiple-temperature BGK scheme with simulations of the inner structures of planar shock waves, one natural requirement is to specify the postshock equilibrium states as the proper initial and downstream boundary conditions. Because of the fact that the downstream flow is always subsonic, the accuracy of the downstream boundary conditions can have significant effects on the simulations.

For a monatomic molecular gas, there are no vibrational and rotational temperatures, and the specific heat ratio $\gamma=C_{p} / C_{v}$ remains constant through shock waves, as long as the postshock temperature is not high enough to trigger an ionization process. Hence, the classical Rankine-Hugoniot relation can provide the correct boundary conditions. However, for diatomic or polyatomic molecules, because the rotational and vibrational degrees of freedom will be activated under a high temperature, the specific heat ratio $\gamma$ will be variant across the shock wave. For this reason, we first proceed to obtain general formulations to compute the postshock equilibrium state for diatomic or polyatomic molecules. At this postshock equilibrium state, we assume that all temperature relaxation processes are completed and that the postshock equilibrium temperature is below that value that is required to trigger severe dissociation.

For a planar shock wave, the equations of density, momentum, and energy are [ㅁ]

$$
\begin{gathered}
\frac{\mathrm{d}}{\mathrm{d} x}(\rho u)=0 ; \quad \frac{\mathrm{d}}{\mathrm{d} x}\left(p+\rho u^{2}-\frac{4}{3} \mu \frac{\mathrm{d} u}{\mathrm{~d} x}\right)=0 \\
\frac{\mathrm{d}}{\mathrm{d} x}\left[\rho u\left(\frac{\gamma}{\gamma-1} R T+\frac{u^{2}}{2}\right)-\frac{4}{3} \mu u \frac{\mathrm{d} u}{\mathrm{~d} x}-\kappa \frac{\mathrm{d} T}{\mathrm{~d} x}\right]=0
\end{gathered}
$$

Select a one-dimensional control volume with one side in the preshock region and the other side in the postshock equilibrium region. Denote the preshock state as state 1 and denote the postshock state, in which all thermal relaxations are complete, as state 2. For these two equilibrium states, we can assume that both satisfy the thermally perfect assumption $p=\rho R T$, where $R_{1}=R_{2}$ with no dissociation effect. Notice that for both states, all gradients are zero. The simple relations between these two states can be obtained as 
follows:

$$
\begin{aligned}
& \frac{T_{2}}{T_{1}}=\frac{\left[\gamma_{1} /\left(\gamma_{1}-1\right)\right]+\left(\gamma_{1} / 2\right) M_{1}^{2}}{\left[\gamma_{2} /\left(\gamma_{2}-1\right)\right]+\left(\gamma_{2} / 2\right) M_{2}^{2}} \\
& \frac{u_{2}}{u_{1}}=\sqrt{\frac{\gamma_{2}}{\gamma_{1}}} \frac{M_{2}}{M_{1}} \sqrt{\frac{\left[\gamma_{1} /\left(\gamma_{1}-1\right)\right]+\left(\gamma_{1} / 2\right) M_{1}^{2}}{\left[\gamma_{2} /\left(\gamma_{1}-1\right)\right]+\left(\gamma_{2} / 2\right) M_{2}^{2}}} \\
& \frac{p_{2}}{p_{1}}=\frac{1+\gamma_{1} M_{1}^{2}}{1+\gamma_{2} M_{2}^{2}} \\
& \left(1+\gamma_{1} M_{1}^{2}\right)^{2} \\
& \overline{\left\{\left[\gamma_{1} /\left(\gamma_{1}-1\right)\right]+\left(\gamma_{1} / 2\right) M_{1}^{2}\right\} \gamma_{1} M_{1}^{2}} \\
& =\frac{\left(1+\gamma_{2} M_{2}^{2}\right)^{2}}{\left\{\left[\gamma_{2} /\left(\gamma_{2}-1\right)\right]+\left(\gamma_{2} / 2\right) M_{2}^{2}\right\} \gamma_{2} M_{2}^{2}}
\end{aligned}
$$

The derivation process is similar to that for the classical RankineHugoniot relation, which can be found in many textbooks, such as those by Saad [9] or Liepmann and Roshko [10]. The preceding equations link the two states with variable specific heat ratios $\gamma_{1}$ and $\gamma_{2}$. We call the preceding equations generalized Rankine-Hugoniot relations across normal shock waves. Because of the relative compactness and general scope of application, the generalized Rankine-Hugoniot relations can be considered as a formulation bounded by the classical shock wave relations without real-gas effects and the relations obtained by by Tisen [11] and Egger [12]. The significance of the preceding relations is evident for equilibrium flows, because they provide a set of postshock state computation formulas. Also, to compute the inner relaxation process across the shock waves with a finite relaxation speed, the preceding equations provide proper downstream boundary conditions.

Although the preceding equations explicitly link the postshock Mach number and the specific heat ratios $\gamma_{2}$, to solve the preceding equations, the expression for the specific heat ratio $\gamma_{2}$ is needed to obtain the postshock state Mach number. To consider the vibrational effects for diatomic and polyatomic molecules with the simple harmonic oscillator model, the specific vibrational energy associated with a mode having a characteristic vibrational temperature $\Theta_{v}$ is [1]

$$
e_{v}=\frac{R \Theta_{v}}{\exp \left(\Theta_{v} / T\right)-1}
$$

The effective number of degrees of freedom at temperature $T$ is [13]

$$
K_{v}=\frac{2 \Theta_{v} / T}{\exp \left(\Theta_{v} / T\right)-1}
$$

For a diatomic molecule and a linear polyatomic molecule, the rotational degree is $K_{r}=2$, and for a nonlinear polyatomic molecule, the rotational degree of freedom is $K_{r}=3$. Then the following iteration process is clear. First, with a specific $\gamma_{2}$, use $M_{1}$ and $\gamma_{1}$ to compute an intermediate postshock Mach number $M_{2}$. Second, use this Mach number $M_{2}$ and the specific heat ratio $\gamma_{2}$ to determine a postshock temperature $T_{2}$. Third, use Eqs. (3) and (19) to determine a new specific heat ratio $\gamma_{2}$. The preceding three steps proceed until an appropriate tolerance is reached.

In the end, we provide some comments to conclude this section:

1) The preceding generalized Rankine-Hugoniot relations consider vibrational temperature effects and are applicable for a wide range of temperatures below dissociation. If the postshock temperature is quite close or higher than the characteristic dissociation temperature, then they are not applicable. If $\gamma_{2}$ is set to $\gamma_{1}$, the preceding relations consistently degenerate to the classical Rankine-Hugoniot relations across planar shock waves.

2) For the hypersonic limit case (i.e., the freestream Mach number is significantly large), $M_{1} \rightarrow \infty$, then

$$
M_{2} \rightarrow \sqrt{\frac{\gamma_{2}-1}{2 \gamma_{2}}} ; \quad \frac{\rho_{1}}{\rho_{2}}=\frac{u_{2}}{u_{1}} \rightarrow \frac{\gamma_{2}-1}{\gamma_{2}+1} ; \quad \frac{T_{2}}{T_{1}} \rightarrow \frac{\gamma_{2}-1}{\gamma_{2}+1} \frac{p_{2}}{p_{1}}
$$

These relations are still compatible with the common results under the hypersonic limit. Note that it is interesting to realize that the postshock equilibrium state has no relations with $\gamma_{1}$; that is, the preshock factor $\gamma_{1}$ is forgotten.

3) Fig. 2 shows two profiles of postshock Mach number with and without this variant specific heat ratio approach. For the constant specific heat ratio case, the specific heat ratio value is set to $\gamma=1.4$. The freestream flow parameters are $T_{\infty}=300 \mathrm{~K}$ and $p_{\infty}=$ $1.10325 \times 10^{5} \mathrm{~Pa}$, and the gas is nitrogen. Figure 3 shows the postshock equilibrium pressure and temperature results vs different freestream Mach numbers, with $T_{\infty}=300 \mathrm{~K}$ and $p_{\infty}=$ $1.01325 \times 10^{5} \mathrm{~Pa}$. It is evident that with the consideration of vibrational temperature or a variable $\gamma$, the postshock equilibrium temperature is lower than the case of constant $\gamma$, whereas the pressure is higher. Hence, the difference in density is well-announced for hypersonic flow when vibrational energy is considered.

4). The derivation process assumes $p=\rho R T$, and the same $R$ is used in the preshock and postshock regions. Hence, when the postshock temperature is close to the characteristic dissociation temperature $\Theta_{d}$, the solution is not applicable because $R$ is not constant. We can use this characteristic dissociation temperature as the upper limit to estimate the asymptote values:

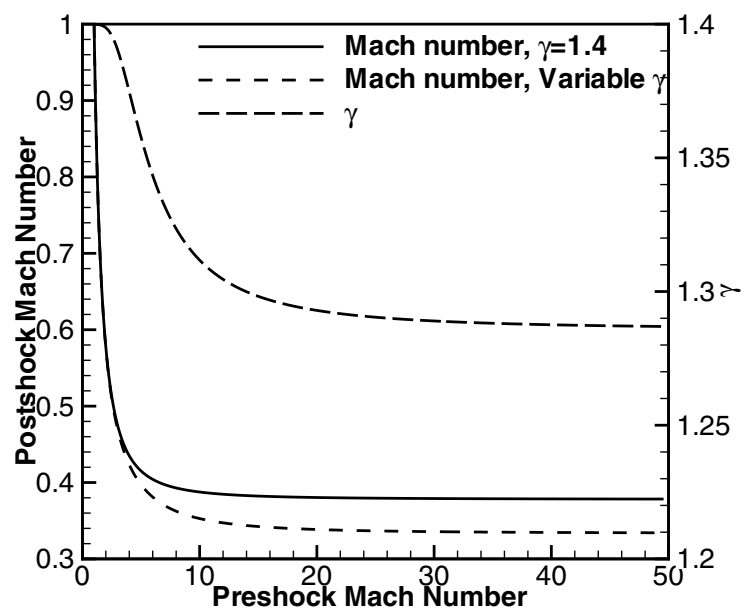

Fig. 2 Profiles of postshock Mach numbers and the specific heat ratio $C_{p} / C_{v}$ (nitrogen, $p_{\infty}=1.01325 \times 10^{5} \mathrm{~Pa}$, and $T_{\infty}=300 \mathrm{~K}$ ).

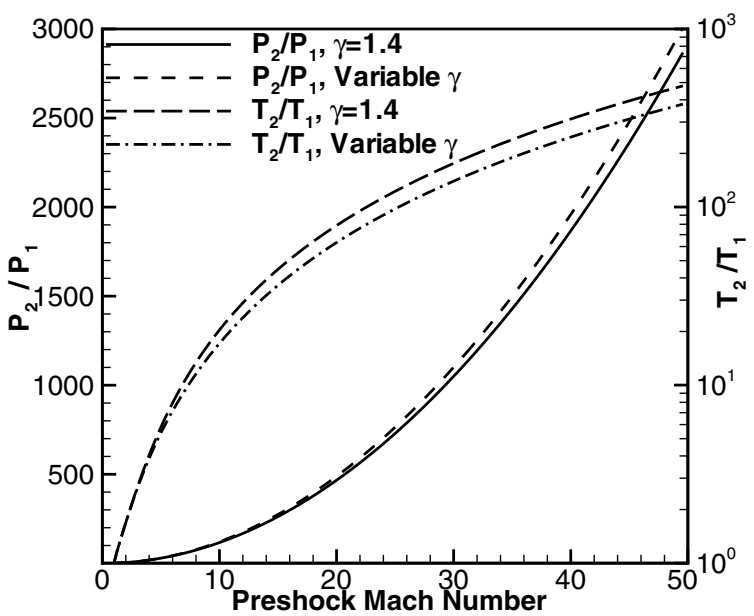

Fig. 3 Profiles of postshock pressure and temperature (nitrogen, $p_{\infty}=1.01325 \times 10^{5} \mathrm{~Pa}$, and $\left.T_{\infty}=300 \mathrm{~K}\right)$. 


$$
K_{v} \rightarrow \frac{2 \Theta_{v} / \Theta_{d}}{\exp \left(\Theta_{v} / \Theta_{d}\right)-1} \simeq \frac{4}{2+\Theta_{v} / \Theta_{d}} ; \quad \gamma \rightarrow \frac{18+7 \Theta_{v} / \Theta_{d}}{14+5 \Theta_{v} / \Theta_{d}}
$$

With the fact that the characteristic vibrational temperature is far less than the dissociation temperature, $\Theta_{v} \ll \Theta_{d}$, the asymptotic solution for the postshock Mach number is

$$
M_{2} \rightarrow \sqrt{\frac{\gamma_{2}-1}{2 \gamma_{2}}}=\sqrt{\frac{2 \Theta_{d}+\Theta_{v}}{18 \Theta_{d}+7 \Theta_{v}}} \rightarrow \frac{1}{3}
$$

This limit value is smaller than the constant specific heat ratio $\sqrt{1 / 7}$ for nitrogen. The physical reason for this $11 \%$ lower value of postshock Mach number for nitrogen is obvious: with the consideration of the vibrational temperature, which has the significantly wider vibrational-energy level, more postshock energy is transferred into the thermal energy. Consequently, the kinetic energy then is significantly lower than is the case in which the vibrational effect is omitted.

5) There are two classical downstream-boundary-condition treatments for the shock wave simulations with the DSMC method [13]. The first treatment is for a planar shock wave with the movingpiston method, which assumes a fixed-equilibrium downstream state described by one unique temperature, and the general RankineHugoniot relations and the harmonic oscillator model can provide the required downstream equilibrium state. The other treatment adopts a stagnation wall treatment, which relaxes the requirement of an equilibrium state for the downstream boundary condition. However, this treatment involves an extra introduction of a stagnation point at the downstream, and all internal temperatures do not necessarily relax to the unique value. Hence, this approach slightly changes the problem from the planar normal shock wave problem in this study. Because of this very reason, we choose the first treatment.

\section{Numerical Simulation and Discussion of Results}

Although there are experimental results of density and translational and rotational temperatures with nitrogen shock waves $[14,15]$, we did not find any corresponding reports with vibrational temperature profiles in the literature. Hence, in this study, we validate the BGK scheme by comparing the numerical results with some DSMC simulation results.

One important issue to discuss first is the rotational- and vibrational-energy relaxation parameters in the DSMC simulations and the kinetic BGK scheme. We used a specific DSMC package named MONACO [16] developed at the University of Michigan. This package is well-tested, and it adopts the nonconstant translational-rotational energy-exchange-probability formula $[17,18]$. As for the translational-vibrational energy-exchange rate $\phi_{\text {tr }- \text { vib }}$, which highly depends on the translational temperature [19], a simple treatment is to use a constant exchange probability for the whole flowfield. For example, in the literature, 0.009 was used for nitrogen gas [19], 0.01 was also used for nitrogen [20], and 0.02 was used for air [21]. In the DSMC simulations with MONACO, the nonconstant translational-rotational energy-exchange rate [19] and fixed translational-vibrational energy-exchange rates used 0.01 or 0.02. Using a smaller value for the translational-vibrational exchange rate will significantly increase the simulation domain and simulation cost. The DSMC simulations are expected to yield fairly accurate results, and we use them as the standard results to compare with those obtained by the gas-kinetic BGK method.

For the gas-kinetic BGK scheme, it is very difficult to accurately determine the parameters $Z_{r}$ and $Z_{v}$ corresponding to the DSMC parameters. Lumpkin et al. [22] pointed out that the particle collision number for the rotational energy should not be the same as the corresponding continuum collision number. We actually feel that more factors should be considered when we attempt to relate the collision numbers for rotational- and vibrational-energy relaxation in the BGK scheme and the DSMC method. First, there are three governing equations in the scheme for translational, rotational, and vibrational energy, whereas in [22], there was only one governing equation. Second, in the scheme, not all of the source terms, which have significant effects on the simulation results, are following the exact Landau-Teller equation format. Third, the adoption of $\tau_{*}$ instead of $\tau$ has significant impacts on the simulation and creates more difference. Finally, the flux evaluation [Eq. (10)], which effectively improves the density-profile smoothness, further increases the difference. Hence, with consideration of the preceding four factors, it is almost impossible to obtain an accurate relation between the rotational collision numbers for the BGK scheme and that for the DSMC method. In the simulations with the BGK scheme, we set $Z_{r}$ to either 3 or 5 , and the vibrational relaxation number is fixed corresponding to the translational-vibrational energyexchange rate in the DSMC method. We believe this fixing of the relaxation numbers is appropriate; otherwise, by freely adjusting them to obtain the best match among the DSMC and BGK simulation results, it is rather unreliable. Here, we want to point out that because the energy redistribution mechanisms are quite different between these two methods, discrepancies are expected with these equivalent relaxation numbers, especially when the vibrational energy is involved and combined with large gradients in the hypersonic flows.

We implemented the preceding gas-kinetic BGK scheme and performed three numerical simulations of strong planar shock waves with the same freestream temperature and density, $T_{\infty}=226.149 \mathrm{~K}$, $\rho_{\infty}=1.7413 \times 10^{-2} \mathrm{~kg} / \mathrm{m}^{3}$, but different freestream Mach numbers: $M_{\infty}=5.0,10.0$, and 15.0. The thermal nonequilibrium effects are significant, and the equilibrium specific heat ratio values are $\gamma_{1}=1.399$ and $\gamma_{2}=1.3713,1.3193$, and 1.3021, correspondingly. In these BGK simulations, the specific relaxation number $Z_{r}$ is set to 3 and $Z_{v}$ is set to 100 for cases I and II, and $Z_{r}$ is set to 5 and $Z_{v}$ is set to 50 for case III. To compare the results, three DSMC simulations are performed for the same cases by using MONACO. To be consistent with the relaxation rate in the BGK scheme, in the DSMC simulations, the translational-vibrational energy-exchange rates are set to 0.01 for cases I and II and 0.02 for case III. The last test case involves a much stronger shock wave, with a postshock equilibrium temperature over $8000 \mathrm{~K}$; hence, an increase for the constant translational-vibrational exchange rate is reasonable. In this study, we concentrate on thermal nonequilibrium flows, and to exclude the complex dissociation effects, the DSMC simulations do not include chemical reactions.

To exclude the differences created by meshes, the same mesh size and mesh number are used by both methods for each case. The cell size is about a tenth of the mean free path based on the freestream flow conditions. With such a fine mesh size, the shock structures in all cases are well-resolved. To properly use the generalized RankineHugoniot relations in the current simulations, we adopted long simulation domains in which the postshock regions have a length of at least a few times that of the shock thickness. To accelerate the simulations for both the BGK and the DSMC simulations, the computational fields are initialized with step profiles in which the postshock quantities are computed with the generalized RankineHugoniot relations instead of the classical Rankine-Hugoniot relations. The BGK scheme uses $\partial() / \partial x=0$ boundary conditions to compute the flux from the downstream boundary, and the specific heat ratio $\gamma$ is recomputed at each time step and for each cell. In the DSMC simulations, the moving-piston scheme [13] is used at the downstream boundary, and a standard shock-stabilization process [13] is adopted as well. In the DSMC simulations, about 1 million particles are used for each simulation case. In all the simulations, the gas is assumed to be nitrogen, even though the current BGK scheme is applicable to simulate shock waves with monatomic gas as well. In the following results, density and temperature profiles are normalized by the values at the two ends of the shocks: for example, $T^{\prime}=\left(T-T_{1}\right) /\left(T_{2}-T_{1}\right)$. In the BGK simulations, the viscosity is computed with a power law formula:

$$
\mu=1.656 \times 10^{-5}(T / 273)^{0.74} \mathrm{~Pa} \cdot \mathrm{s}
$$

Correspondingly, the variable hard sphere model [13] was used in the DSMC simulation with a viscosity index factor $\omega=0.74$. 
Figures 4-7 show the simulation results for the first test case with the DSMC method and the BGK scheme. Figure 4 shows the density profiles, Fig. $\underline{5}$ shows the translational temperature profiles, Fig. $\underline{6}$ shows the rotational-temperature profiles, and Fig. 7 shows the vibrational temperature and the specific heat ratio profiles. It is quite evident that the thermal nonequilibrium effects and the relaxation processes are clearly captured and that the results are fairly close. Remarkably, the density profile is quite smooth. It has been generally believed that the DSMC method is accurate for many rarefied flow simulations, and it yields very accurate inner structures for shock waves. For gas-kinetic CFD schemes, it is generally very difficult to provide close inner shock structures, especially for density profiles [23]. Obviously, the BGK scheme in this study has a relatively better performance on such a difficult problem. We believe the reasons are due to the different numerical flux evaluation formula, the flux average with $f_{\text {eq }}$ [Eq. (10)], and, especially, the adoption of the $\tau_{*}$ treatment. Almost all of the results in these figures show good matches, whereas the vibrational temperature results have the largest discrepancy at the preshock region. The DSMC simulation results predict higher values for the vibrational temperature at the preshock region. We want to point out that this was not physically observed in experiments and this discrepancy is generated by the selection of 0.01 for the translational-vibrational energy-exchange rate. This actual rate highly depends on the translational temperature [24]. With a freestream temperature $300 \mathrm{~K}$, the local relaxation number should

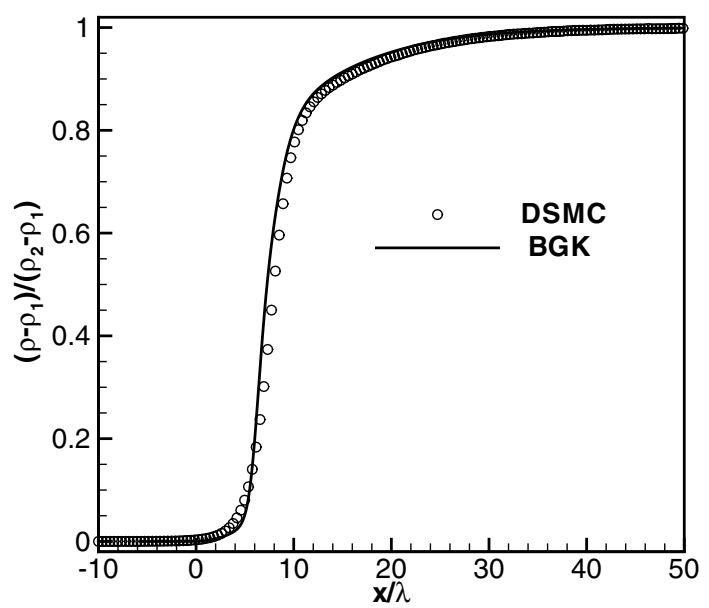

Fig. 4 Test case $I$ : density profiles in an $M=5.0$ nitrogen shock wave $\left(Z_{\mathrm{vib}, \mathrm{BGK}}=100, \quad Z_{\mathrm{rot}, \mathrm{BGK}}=3, \quad \phi_{\text {tr-vib }}=0.01, \quad \rho_{\infty}=1.741315 \mathrm{x}\right.$ $\left.10^{-2} \mathrm{~kg} / \mathrm{m}^{3}, T_{\infty}=226.649 \mathrm{~K}\right)$.

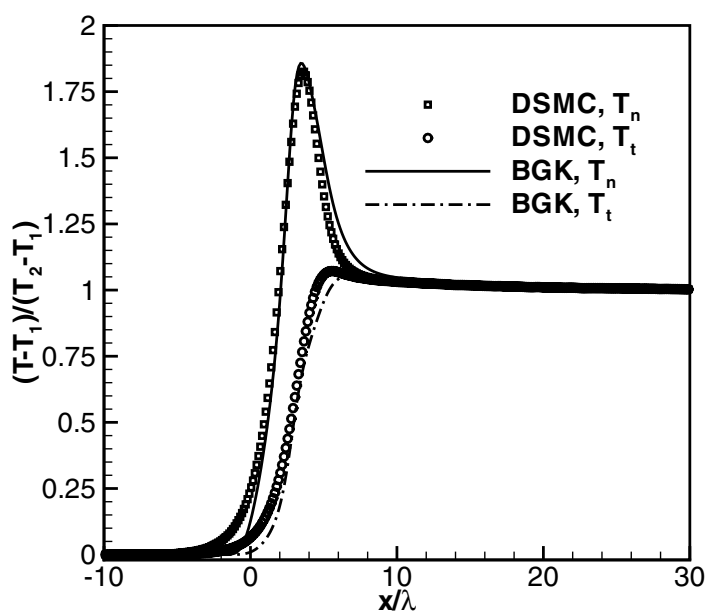

Fig. 5 Test case $I$ : translational temperature profiles in an $M=\mathbf{5 . 0}$ nitrogen shock wave $\left(Z_{\mathrm{vib}, \mathrm{BGK}}=100, Z_{\mathrm{rot}, \mathrm{BGK}}=3, \phi_{\text {tr-vib }}=0.01\right.$, $\rho_{\infty}=1.741315 \times 10^{-2} \mathrm{~kg} / \mathrm{m}^{3}$, and $\left.T_{\infty}=226.649 \mathrm{~K}\right)$.

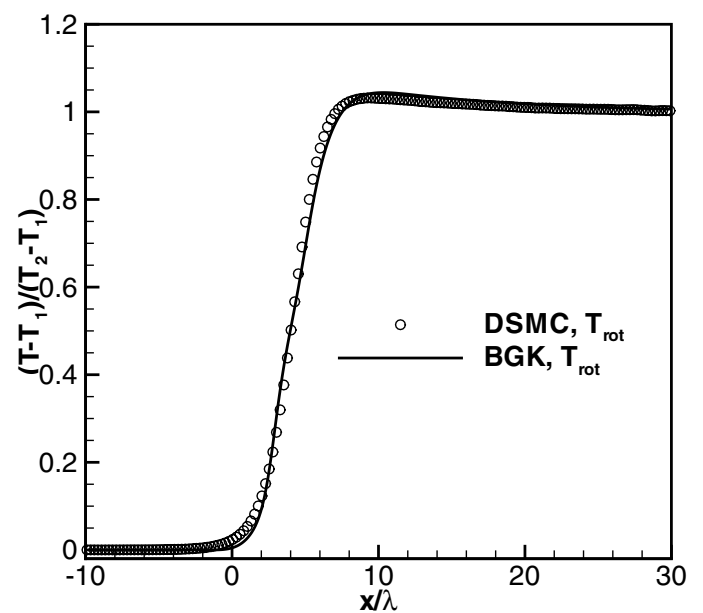

Fig. 6 Test case I: rotational temperature profile in an $M=5.0$ nitrogen shock wave $\left(Z_{\mathrm{vib}, \mathrm{BGK}}=100, Z_{\mathrm{rot}, \mathrm{BGK}}=3, \phi_{\text {tr-vib }}=0.01\right.$, $\rho_{\infty}=1.741315 \times 10^{-2} \mathrm{~kg} / \mathrm{m}^{3}$, and $\left.T_{\infty}=226.649 \mathrm{~K}\right)$.

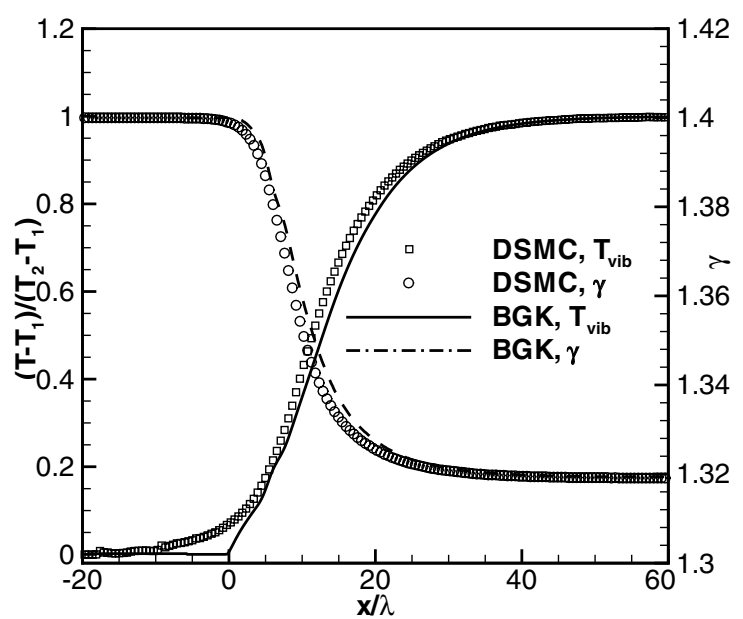

Fig. 7 Test case I: vibrational temperature and $\gamma$ profiles in an $M=\mathbf{5 . 0}$ nitrogen shock wave $\left(Z_{\mathrm{vib}, \mathrm{BGK}}=100, Z_{\mathrm{rot}, \mathrm{BGK}}=3, \phi_{\text {tr-vib }}=0.01\right.$, $\rho_{\infty}=1.741315 \times 10^{-2} \mathrm{~kg} / \mathrm{m}^{3}$, and $\left.T_{\infty}=226.649 \mathrm{~K}\right)$.

be less than $1 \times 10^{-5}$. This value increases significantly across the shock wave when the translational temperature increases. With a high, unrealistic, rate of 0.01 , some particles inside the shock acquire abnormally high vibrational energy from the translational energy, because the jump between the quantum-vibrational-energy level is much wider than that for either a rotational- or translational-energylevel difference. When some of the particles with high vibrational energy diffuse back from the inner layer of the shock wave to the front side of the shock, due to the slow-vibrational-energy relaxation rate, a false high-vibrational-energy distribution in front of the shock is formed. Although there are particles with high rotational energy that can diffuse back to the preshock regime as well, because the rotational energy level is much narrower and the rotational-energy relaxation rate is fast, the DSMC results for the rotational temperature quickly drops to the preshock level. To confirm this conjecture, we performed another simulation with a smaller translational-vibrational energy-exchange rate of 0.001 , and the abnormal increase of vibrational temperature at the preshock region decreased significantly. However, the penalty for adopting such a small value of 0.001 was a significantly long simulation domain and more expensive simulation cost. Meanwhile, this defect in the DSMC simulation results for the vibrational temperature in the preshock region is not quite serious, for two reasons: First, the good matches among the density and other temperature profiles from the BGK and DSMC simulation data, even the other portion of the vibrational temperature, support the validity of the BGK scheme. 
Second, the vibrational energy in front of the shock possesses almost zero percentage of the total energy, even though at the equilibrium state of the postshock region, the vibrational energy only possess $7 \%$ of the total inner energy for the first test case. Hence, although the vibrational temperature at the preshock region has the largest discrepancies among all of the simulation results, its effects on the total inner energy budget are quite limited.

Figures $8-10$ show the corresponding results for the case $M=10.0$, and Figs. $11-13$ show the results for case $M=15.0$. The preceding analysis holds for these two test cases with strong shocks as well. The results for the last case, with the strongest shock wave, show relatively larger discrepancies, which are expected. For the last case, a single value for the translational-vibrational energyexchange probability seems inadequate for the stronger shock gradients and the wider translational temperature scope. Another reason is that to maintain simplicity, the complex dissociationrecombination mechanism is not included for both the DSMC method and the BGK scheme. This simplification results in some degree of nonphysical results in the last case. The mission of this study is to test the newly developed BGK scheme for thermal nonequilibrium flows, and the last test case is used to demonstrate the application scope. A new BGK scheme with a consideration of chemically reacting flows is currently near completion.

After preparing this paper, we noticed two recent papers on gaskinetic CFD schemes: $[25,26]$. Readers are encouraged to read these two papers and may find them very interesting.

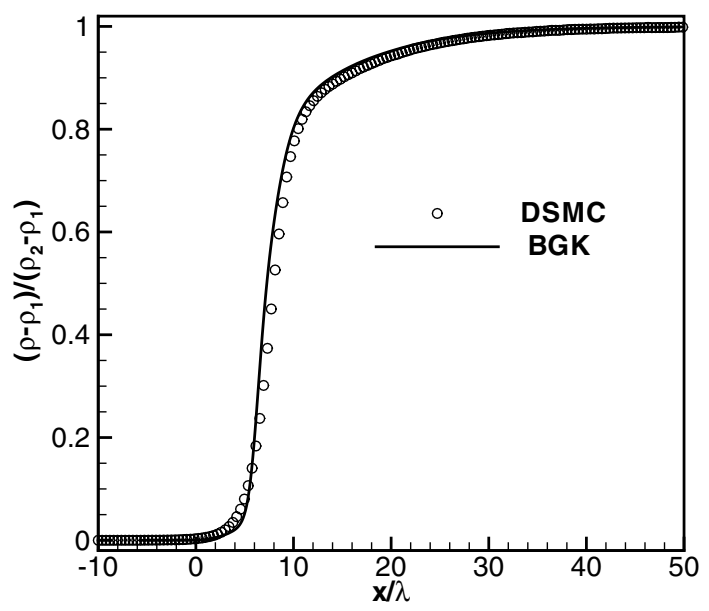

Fig. 8 Test case II: density profiles in an $M=10.0$ nitrogen shock wave $\left(Z_{\mathrm{vib}, \mathrm{BGK}}=100, \quad Z_{\text {rot }, \mathrm{BGK}}=3, \quad \phi_{\text {tr-vib }}=0.01, \quad \rho_{\infty}=1.741315 \mathrm{x}\right.$ $10^{-2} \mathrm{~kg} / \mathrm{m}^{3}$, and $\left.T_{\infty}=226.649 \mathrm{~K}\right)$.

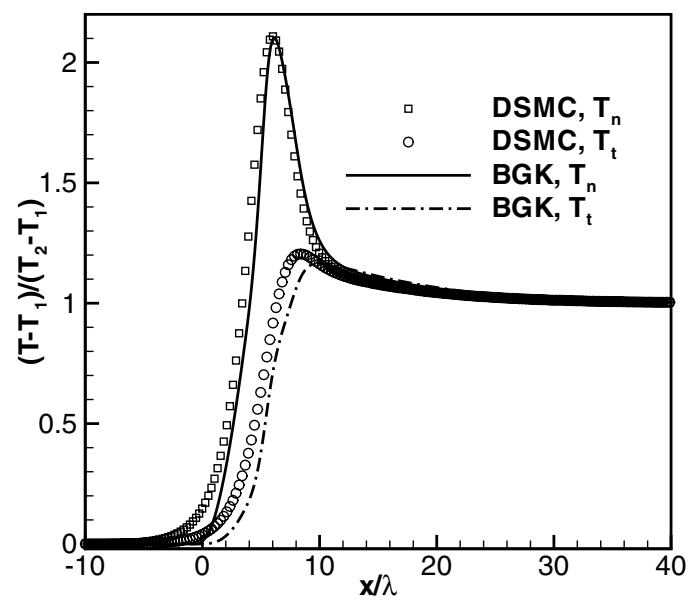

Fig. 9 Test case II: translational temperature profiles in an $M=\mathbf{1 0 . 0}$ nitrogen shock wave $\left(Z_{\text {vib,BGK }}=100, Z_{\text {rot }, \mathrm{BGK}}=3, \phi_{\text {tr-vib }}=0.01\right.$, $\rho_{\infty}=1.741315 \times 10^{-2} \mathrm{~kg} / \mathrm{m}^{3}$, and $\left.T_{\infty}=226.649 \mathrm{~K}\right)$.

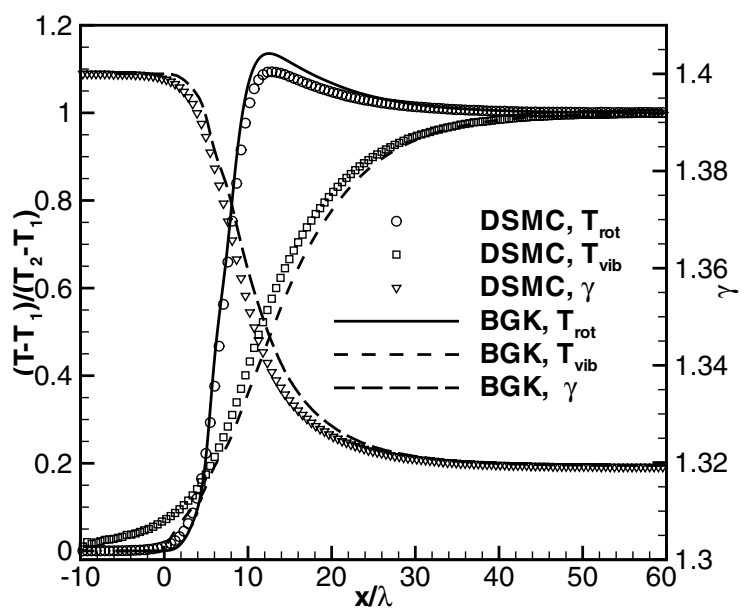

Fig. 10 Test case II: rotational-vibrational temperature and $\gamma$ profiles in an $M=10.0$ nitrogen shock wave $\left(Z_{\mathrm{vib}, \mathrm{BGK}}=100, Z_{\mathrm{rot}, \mathrm{BGK}}=3\right.$, $\phi_{\text {tr-vib }}=0.01, \rho_{\infty}=1.741315 \times 10^{-2} \mathrm{~kg} / \mathrm{m}^{3}$, and $\left.T_{\infty}=226.649 \mathrm{~K}\right)$.

\section{Conclusions}

In this paper, we reported some recent progress in developing a one-dimensional thermal nonequilibrium gas-kinetic BGK scheme. This BGK scheme includes the vibrational-energy effects, and it can

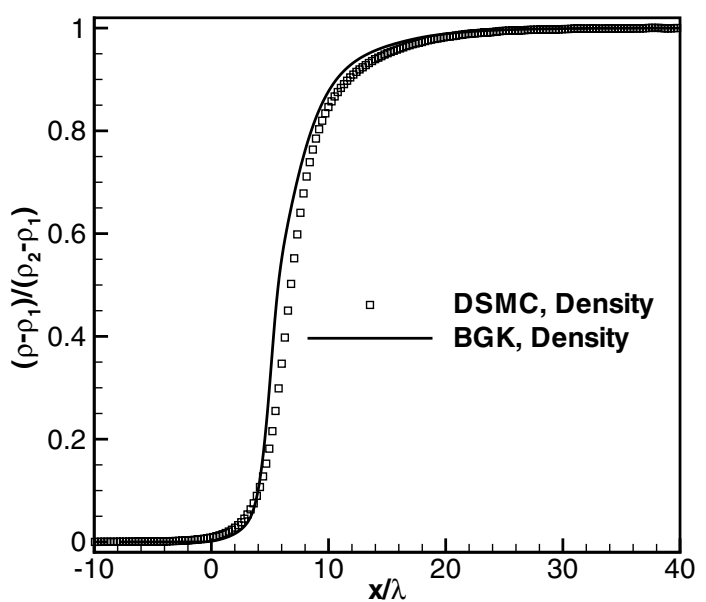

Fig. 11 Test case III: density profiles in an $M=15.0$ nitrogen shock wave $\left(Z_{\text {vib,BGK }}=50, Z_{\text {rot, }, \mathrm{BGK}}=5, \phi_{\text {tr-vib }}=0.02, \rho_{\infty}=1.741315 \mathrm{x}\right.$ $10^{-2} \mathrm{~kg} / \mathrm{m}^{3}$, and $\left.T_{\infty}=226.649 \mathrm{~K}\right)$.

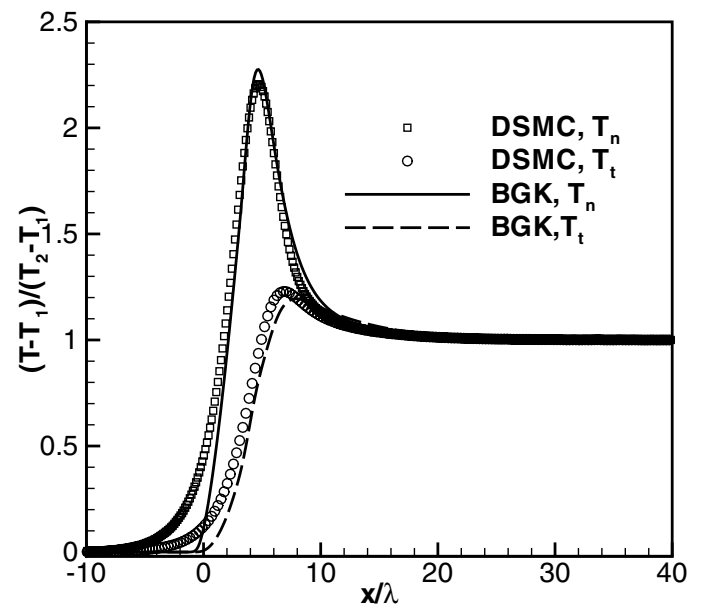

Fig. 12 Test case III: translational temperature profiles in an $M=\mathbf{1 5 . 0}$ nitrogen shock wave $\left(Z_{\mathrm{vib}, \mathrm{BGK}}=50, Z_{\mathrm{rot}, \mathrm{BGK}}=5, \phi_{\mathrm{tr}-\mathrm{vib}}=0.02\right.$, $\rho_{\infty}=1.741315 \times 10^{-2} \mathrm{~kg} / \mathrm{m}^{3}$, and $\left.T_{\infty}=226.649 \mathrm{~K}\right)$. 


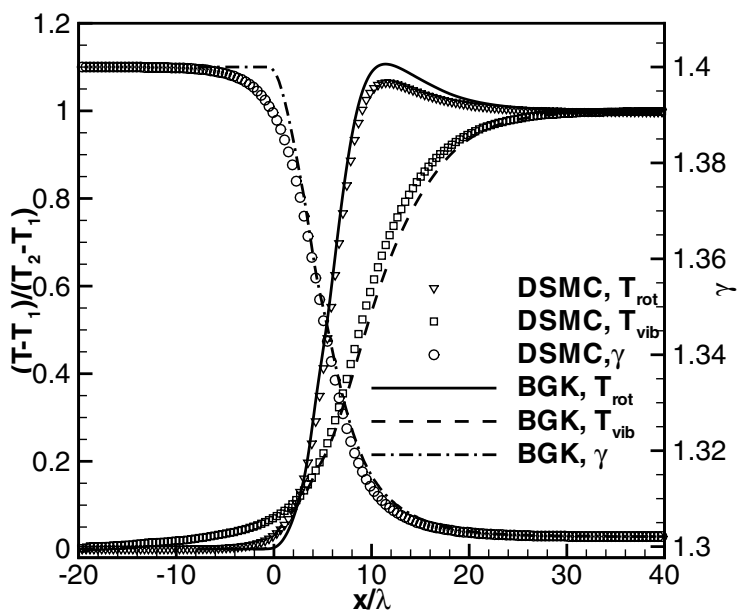

Fig. 13 Test case III: rotational-vibrational temperature and $\gamma$ profiles in an $M=15.0$ nitrogen shock wave $\left(Z_{\mathrm{vib}, \mathrm{BGK}}=50, Z_{\mathrm{rot}, \mathrm{BGK}}=5\right.$, $\phi_{\text {tr-vib }}=0.02, \rho_{\infty}=1.741315 \times 10^{-2} \mathrm{~kg} / \mathrm{m}^{3}$, and $\left.T_{\infty}=226.649 \mathrm{~K}\right)$.

be used in simulating the inner structures of strong planar shock waves. Compared with the DSMC method, this scheme is capable of reducing the simulation cost significantly. The shock wave computation is also stable during the computation. A set of generalized Rankine-Hugoniot relations were obtained and used to provide the downstream boundary conditions for both the DSMC method and the BGK scheme. Numerical simulations of strong shock waves indicated that the BGK scheme could capture the inner structures for the shock waves with vibrational-energy effects, and the profiles are quite smooth and close to the DSMC results.

This development sets a solid foundation for the constructions of general two-dimensional and three-dimensional gas-kinetic BGK schemes for thermal nonequilibrium flows.

\section{Appendix: Determination of Spatial Gradients for the Equilibrium Distribution Function}

The equilibrium state of gas can be expressed as

$$
\begin{aligned}
g= & \rho\left(\frac{\lambda_{x}}{\pi}\right)^{1 / 2} \exp \left(-\lambda_{x}(u-U)^{2}\right)\left(\frac{\lambda_{t}}{\pi}\right)^{K_{t} / 2} \\
& \times \exp \left(-\lambda_{t} \xi_{t}^{2}\right)\left(\frac{\lambda_{r}}{\pi}\right)^{K_{r} / 2} \exp \left(-\lambda_{r} \xi_{r}^{2}\right)\left(\frac{\lambda_{v}}{\pi}\right)^{K_{v} / 2} \exp \left(-\lambda_{v} \xi_{v}^{2}\right)
\end{aligned}
$$

where $\xi_{t}, \xi_{r}$, and $\xi_{v}$ have $K_{t}, K_{r}$, and $K_{v}$ degrees of freedom of translational, rotational, and vibrational energy.

Because

$$
\left(\rho, \rho U, \rho E, \rho E_{t}, \rho E_{r}, \rho E_{v}\right)^{T}=\int g \Psi \mathrm{d} u \mathrm{~d} \xi_{t} \mathrm{~d} \xi_{r} \mathrm{~d} \xi_{v}
$$

where $\rho E, \rho E_{t}, \rho E_{r}$ and $\rho E_{v}$ are the total, translational, rotational, and vibration energy, correspondingly, and

$$
\Psi=\left\{1, u, \frac{1}{2}\left(u^{2}+\xi_{t}^{2}+\xi_{r}^{2}+\xi_{v}^{2}\right), \frac{1}{2} \xi_{t}^{2}, \frac{1}{2} \xi_{r}^{2}, \frac{1}{2} \xi_{v}^{2}\right\}^{T}
$$

By the Taylor expansion,

$$
\frac{\partial g}{\partial x}=\left(a_{1}+a_{2} u+a_{3} u^{2}+a_{4} \xi_{t}^{2}+a_{5} \xi_{r}^{2}+a_{6} \xi_{v}^{2}\right) g
$$

and

$$
\frac{\partial}{\partial x}\left(\rho, \rho U, \rho E, \rho E_{t}, \rho E_{r}, \rho E_{v}\right)^{T}=\int \frac{\partial g}{\partial x} \Psi \mathrm{d} u \mathrm{~d} \xi_{t} \mathrm{~d} \xi_{r} \mathrm{~d} \xi_{v}
$$

The preceding equations have the following solutions:

$$
\begin{gathered}
a_{6}=\frac{4 \lambda_{v}^{2}}{K_{v}} \frac{\partial\left(\rho E_{v}\right)}{\rho \partial x}-\frac{\partial \rho}{\rho \partial x} \lambda_{v} ; \quad a_{5}=\frac{4 \lambda_{r}^{2}}{K_{r}} \frac{\partial\left(\rho E_{r}\right)}{\rho \partial x}-\frac{\partial \rho}{\rho \partial x} \lambda_{r} \\
a_{4}=\frac{4 \lambda_{t}^{2}}{K_{t}} \frac{\partial\left(\rho E_{t}\right)}{\rho \partial x}-\frac{\partial \rho}{\rho \partial x} \lambda_{t}
\end{gathered}
$$

Further define

$$
\begin{aligned}
A= & \frac{\partial \rho}{\rho \partial x}-\frac{K_{v}}{2 \lambda_{v}} a_{6}-\frac{K_{r}}{2 \lambda_{r}} a_{5}-\frac{K_{t}}{2 \lambda_{t}} a_{4} ; \quad B=\frac{\partial(\rho U)}{\rho \partial x}-\frac{\partial \rho}{\rho \partial x} U \\
C & =\frac{\partial(\rho E)}{\rho \partial x}-\frac{\partial\left(\rho E_{t}\right)}{\rho \partial x}-\frac{\partial\left(\rho E_{r}\right)}{\rho \partial x}-\frac{\partial\left(\rho E_{v}\right)}{\rho \partial x} \\
& -\frac{1}{4}\left(U^{2}+\frac{1}{2 \lambda_{x}}\right)\left(\frac{K_{t} a_{4}}{\lambda_{t}}+\frac{K_{r} a_{5}}{\lambda_{r}}+\frac{K_{v} a_{6}}{\lambda_{v}}\right)-\frac{A}{2}\left(U^{2}+\frac{1}{2 \lambda_{x}}\right)
\end{aligned}
$$

Then

$$
\begin{gathered}
a_{3}=4 \lambda_{x}^{2}[C-B U] ; \quad a_{2}=2 \lambda_{x}\left[B-\frac{U a_{3}}{\lambda_{x}}\right] \\
a_{1}=A-\left(U^{2}+\frac{1}{2 \lambda_{x}}\right) a_{3}-U a_{2}
\end{gathered}
$$

\section{Acknowledgments}

This work is supported by the U.S. Air Force Office of Scientific Research contract FA9550-06-C-0003, with tasks monitored by Jonathan Poggie and John Schmisseur. The authors thank Iain D. Boyd for his comments on the rotational and vibrational collision numbers and Quanhua Sun for his help on the simulations of shock waves with the direct simulation Monte Carlo method. We also extend our sincere thanks to the reviewers, whose valuable suggestions significantly improved the quality of this paper.

\section{References}

[1] Aristov, V. V., Direct Methods for Solving the Boltzmann Equation and Study of Non-Equilibrium Flows, Kluwer Academic, Norwell, MA, 2001.

[2] Xu, K., and Josyula, E., "Multiple Translational Temperature Model and its Shock Structure Solution," Physical Review E (Statistical Physics, Plasmas, Fluids, and Related Interdisciplinary Topics), Vol. 71, No. 5, 2005, pp. 056-308.

[3] Xu, K., "Non-Equilibrium Bhatnagar-Gross-Krook Model for Nitrogen Shock Structure," Physics of Fluids, Vol. 16, No. 10, 2004, 3824-3827.

[4] Xu, K., "A Gas-Kinetic BGK Scheme for the Navier-Stokes Equations, and Its Connection with Artificial Dissipation and Godunov Method," Journal of Computational Physics, Vol. 171, No. 1, July 2001, pp. 289335 . doi:10.1006/jcph.2001.6790

[5] Kogan, K. N., Rarefied Gas Dynamics, Plenum, New York, 1969.

[6] Xu, K., Mao, M., and Tang, L., "A Multidimensional Gas-Kinetic BGK Scheme for Hypersonic Viscous Flow," Journal of Computational Physics, Vol. 203, No. 2, 2005, pp. 405-421.

[7] $\mathrm{Xu}, \mathrm{K}$., "Regulation of the Chapman-Enskog Expansion and Its Description of Shock Structure," Physics of Fluids, Vol. 14, No. 4, 2002, 17-20.

[8] Zel'dovich, Ya. B., and Raizer, Yu. P., Physics of Shock Waves and High-Temperature Hydrodynamic Phenomena, edited by W. D. Hayes, and R. F. Probstein, Dover, New York, 2002.

[9] Saad, M. A., Compressible Fluid Flow, Prentice-Hall, Englewood Cliffs, NJ, 1993.

[10] Liepmann, H. W., and Roshko, A., Elements of Gasdynamics, Wiley, New York, 1957.

[11] Tisen, H. S., "One-Dimensional Flows of a Gas Characterized by Van Der Waal's Equation of State," Journal of Mathematical Physics (Cambridge, Mass.), Vol. 26, No. 6, Jan. 1947, pp. 301-324. 
[12] Eggers, A. J., Jr., "One Dimensional Flows of an Imperfect Diatomic Gas,” NACA Rept. 959, 1950.

[13] Bird, G. A., Molecular Gas Dynamics and the Direct Simulation of Gas Flows, Clarendon, Oxford, 1994.

[14] Aslmeyer, H., "Density Profiles in Argon and Nitrogen Shock Waves Measured by the Absorption of an Electron Beam," Journal of Fluid Mechanics, Vol. 74, No. 3, 1976, pp. 497-513. doi:10.1017/S0022112076001912

[15] Robben, F., and Talbot, L., "Experimental Study of the Rotational Distribution Function of Nitrogen in a Shock Wave," Physics of Fluids, Vol. 9, No. 4, 1966, pp. 653-662. doi:10.1063/1.1761730

[16] Dietrich, S., and Boyd, I. D., "Scalar and Parallel Optimized Implementation of the Direct Simulation Monte Carlo Method," Journal of Computational Physics, Vol. 126, No. 2, 1996, pp. 328-342. doi:10.1006/jcph.1996.0141

[17] Boyd, I. D., "Rotational-Translational Energy Transfer in Rarefied Non-Equilibrium Flows," Physics of Fluids A, Vol. 2, No. 3, 1990, pp. $447-452$ doi:10.1063/1.857740

[18] Boyd, I. D., "Analysis of Rotational Non-Equilibirum in Standing Shock Waves of Nitrogen," AIAA Journal, Vol. 28, No. 11, 1990, 1997-1999.

[19] Boyd, I. D., "Analysis of Vibrational-Translational Energy Transfer Using the Direct Simulation Monte Carlo Method," Physics of Fluids A, Vol. 3, No. 7, 1991, pp. 1785-1791. doi:10.1063/1.857959

[20] Bird, G. A., "Direct Molecular Simulation of a Dissociating Diatomic Gas," Journal of Computational Physics, Vol. 25,
Dec. 1977 , pp. 353-365.

doi:10.1016/0021-9991(77)90003-1

[21] Moss, J. N., and Bird, G. A., "Direct Simulation of Transitional Flow for Hypersonic Reentry Conditions," 22nd AIAA Aerospace Sciences Meeting, Reno, NV, AIAA Paper 1984-223, Jan. 1984.

[22] Lumpkin, F. E., Hass, B. L., and Boyd, I. D., "Resolution of Differences Between Collision Number Definitions in Particle and Continuum Simulations," Physics of Fluids A, Vol. 3, No. 9, Sept. 1991, pp. 22822284. doi: $10.1063 / 1.857964$

[23] Candler, G., Nijhawan, S., Bose, D., and Boyd, I. D., "A Multiple Translational Temperation Gas Dynamics Model," Physics of Fluids, Vol. 6, No. 11, 1994, pp. 3776-3786. doi: $10.1063 / 1.868367$

[24] Parker, J. G., "Rotational and Vibrational Relaxation in Diatomic Gases," Physics of Fluids, Vol. 2, No. 4, July-Aug. 1959, pp. 449-462. doi:10.1063/1.1724417

[25] Agarwal, R., and Chen, R., "Computation of Hypersonic Shock Wave Flows of Diatomic Gases Using the Generalized Boltzmann Equation," 39th AIAA Thermophysics Conference, Miami, FL, AIAA Paper 20074541, 25-28th June 2007.

[26] Arslanbekov, R, R., Kolobov, V. I., Frolova, A., Zabelok, S., and Josyula, E., "Evaluation of a Unified Kinetic/Continuum Solver for Computing Heat Flux in Hypersonic Blunt Body Flows," 39th AIAA Thermophysics Conference, Miami, FL, AIAA, Paper 2007-4544, June 2007. 